\title{
CONCEPTOS FUNDAMENTALES DEL CRISTIANISMO DE KIERKEGAARD, A 200 AÑOS DE SU NACIMIENTO
}

\author{
Matías Tapia Wende \\ Magíster en Filosofía \\ Universidad de Chile \\ mtapiawende@gmail.com
}

\begin{abstract}
Resumen / Abstract
Este artículo tiene como objetivo clarificar algunas nociones fundamentales de la reconstrucción del cristianismo de Søren Kierkegaard (1813-1855). En primer lugar, se hará una distinción entre creencia histórica y fe cristiana, que sirve de base para entender la relación existencial que el individuo puede desarrollar con la enseñanza de Cristo. Seguidamente, se hará alusión al concepto de espíritu que propone el pensador danés, demarcando con esto los estadios kierkegaardianos de la existencia. Y, por último, se conectarán ambos puntos anteriores con lo que entiende Kierkegaard por verdad y libertad en el contexto religioso.
\end{abstract}

Palabras clave: Søren Kierkegaard, cristianismo, fe, espíritu, verdad, libertad.

FuNDAMENTAL CONCEPTS OF KIERKEGAARD'S CHRISTIANITY, 200 YEARS AFTER ITS BIRTH

This article aims to clarify some fundamental notions of the Christianity's reconstruction of Søren Kierkegaard (1813-1855). First, there will be a distinction between historical belief and Christian faith, which serves as a basis for understanding the existential relationship that the individual can develop with the teaching of Christ. Thereafter, there will be a reference to the concept of spirit proposed by the Danish thinker, marking with this the Kierkegaardian stages of existence. And finally, the two points above will be connected with what Kierkegaard means by truth and freedom in the religious context.

KEY WORDS: Søren Kierkegaard, Christianity, faith, spirit, truth, freedom.

\section{Cristianismo en la cristiandad}

RA Hacia el final de su vida, el 28 de marzo de 1855, Kierkegaard escribe un artículo en el diario danés Fcedrelandet, titulado "Una tesis - solo una" ("En Thesis -kun een eneste'), que comienza diciendo: “Oh, Lutero, tú tenías 95 tesis -iterrible! Sin embargo, en un sentido más profundo, mientras más tesis existan, menos terrible es la situación. Este caso es muchísimo más terrible, 1855: hay solo una tesis. El cristianismo del Nuevo Testamento simplemente no existe" (SKS 14, 
p. 169; las cursivas son nuestras) ${ }^{1}$. Esta crítica, que aparece solo cuatro días después del primer número de la revista que el mismo Kierkegaard fundara para atacar a la cristiandad reinante en Dinamarca, llamada El instante (Øieblikket), está inmersa en una efusiva serie de publicaciones que marcaron el último periodo, y el más radical, de este pensador.

En la base de estas arremetidas está lo que él cree es su deber providencial, esto es, volver a "introducir el cristianismo en la cristiandad" (Pap. X, 1 A 138), pues el mensaje de Cristo no es para grandes multitudes, sino para el individuo singular y apartado $^{2}$, que delante de Dios es capaz de hacer suya la imagen del Mesías y vivir de forma imitativa: el cristianismo es una comunicación existencial, es el modelo para saber cómo existir siendo humano y, por ende, es la única solución consistente de la existencia individual (PS, p. 373 / SKS 7, p. 346). Asimismo, es a su vez una labor que envuelve la existencia toda, por lo que no habrá vida cristiana que no sea camino ni devenir constantes, ni cristianismo que no exprese la verdad de la eternidad encarnada en el tiempo en la medida en que lo permitan las fuerzas del existente; todo lo que sea distinto a esto es paganismo para Kierkegaard (PS, p. 28 / SKS 7, p. 25).

En la misma línea, como el cristianismo debe ser individual y constante, debe ser también consciente. No hay quien nazca siendo un auténtico cristiano ni hay quien llegue a serlo sin antes haber reflexionado profundamente sobre su interioridad. La verdadera conversión, que es aquella que cambia radicalmente la existencia del hombre singular, llega recién cuando este se encuentra en su madurez, así como el cristianismo, históricamente hablando, no llegó sino cuando la humanidad había ya salido de su infancia reflexiva (PS, p. 582 / SKS, 7, pp. 545-546).

Estas categorías de la individualidad, la constancia y la conciencia cristianas, aplicadas a la fe, al espíritu, a la verdad y a la libertad, son lo que desarrollaremos en lo sucesivo.

1 La citación de los textos de Kierkegaard se hará apuntando la edición castellana, si estuviere disponible, abreviada con su sigla correspondiente (el método de abreviación se indicará en la bibliografía sumaria) y su número de página. Asimismo, se dará la referencia de las Obras Completas con la sigla SKS (Søren Kierkegaards Skrifter), seguida del tomo y la página. En el caso de sus papeles personales o Papirer, cuya edición más extensa encontrada fue la alemana, se citarán según número de volumen, grupo (A, B o C) y número de entrada, anteponiendo la abreviación Pap. Si no se indica versión española alguna tanto en las obras, como en sus papeles, la traducción es nuestra.

2 A lo largo de este artículo, no se verá delimitación entre individuo, hombre singular, ser humano, yo, ser sí mismo, etc. Esto, porque en Kierkegaard todas estas nociones vienen a significar lo mismo, al menos en el contexto religioso (Cfr. Binneti, 2005, p. 9, en nota). 


\section{Creencia histórica y fe cristiana}

Kierkegaard, a diferencia de la tradición alemana contemporánea a él, que comienza Feuerbach y que continúa Marx, concibe el cristianismo como un hecho histórico, es decir, afirma que esta doctrina se presentó hace poco más de dos mil años en la figura de un hombre de carne y hueso llamado Cristo, que vivió y que murió tal como lo haría cualquier otro hombre (PS, p. 559 / SKS 7, p. 526). Esta forma de comunicación divina no es al azar, sino que supone una consideración sobremanera atenta de Dios para acercarse a la raza humana en el parámetro esencial que la delimita: la existencia. Por lo tanto, la relación de cualquier individuo con el cristianismo será, de una u otra forma, similar a la que se da con cualquier otro suceso que haya irrumpido en la historia. Sin embargo, falta todavía una distinción de grado que resulta capital.

La visión kierkegaardiana de la historia comprende cualquier hecho histórico como meramente contingente, pues lo que determina la ocurrencia de tal o cual suceso es el devenir, y en nada que devenga hallamos necesidad, sino solo incertidumbre (MF, p. 86 / SKS 4, p. 278). Esto nos vuelve a la concepción tenida por Leibniz de que las verdades de hecho son pura contingencia, a diferencia de las verdades de razón que son esenciales y necesarias (Gaye 1987, pp. 105-109). Sabemos que César cruzó el Rubicón y que Napoleón entró a Berlín por la Puerta de Brandemburgo, pero no hay nada que estrictamente nos lleve a pensar que la situación opuesta a ambos sucesos no sea concebible. Teniendo en cuenta, entonces, que nada en la historia es necesario, la única forma que tiene el individuo para relacionarse con lo histórico es a través de una voluntad de creer, que anula las dudas que genera lo contingente y crea para el individuo la imagen de un vínculo parecido al de contemporaneidad ${ }^{3}$. Así se trata este asunto cuando está en juego lo meramente histórico.

Por otro lado, todo cambia radicalmente con el cristianismo. Lo que ahora exige creencia no es un algo contingente que llega a devenir, sino que es lo eternamente necesario que se somete al desarrollo del tiempo: Dios se hace carne en Cristo, y lo que ha sido por toda la eternidad, de manera infinita, ingénita e imperecedera, se presenta ahora en forma humana. Ya no basta tener una creencia histórica, sino que se exige de plano la fe en tanto don de gracia ${ }^{4}$. La figura de Cristo como el Dios-Hombre es lo que repele esencialmente a la razón y por lo mismo necesita de algo externo, que venga

3 Para Kierkegaard, la existencia que es contemporánea con un cierto hecho histórico es la única que no exige pruebas de ningún tipo en relación con éste. Por ejemplo, si a mi lado se encuentra un hombre que me asegura que existe, yo de buena gana le confirmo su afirmación. Pero si, acto seguido, este mismo hombre me asevera a pie juntillas que tiene doscientos años, ya no es algo que competa a mi contemporaneidad, sino a mi creencia.

$4 \quad$ Esta diferencia se hace de manera externa al pensamiento de Kierkegaard, pero se deriva de éste con mucha claridad. El único elemento que puede llevar a confusión es que el vocablo danés Tro es el que utiliza este pensador para designar indistintamente a una o a otra creencia, ya sea a la que es tal en sentido ordinario o a la que es tal en sentido absoluto. En alemán el problema es el mismo, pues Glaube existe para ambos conceptos. En inglés, 
desde arriba, y que suprima la paradoja absoluta que representa que la divinidad se encalle ahora en inmanencia. Este elemento exotérico es la condición (Betingelsen) de la fe, la feliz pasión que surge como un tercero después que la razón conscientemente se hace a un lado y la paradoja se abandona a sí misma en su incomprensión (MF, p. 70 / SKS 4, p. 261). Aquí no es suficiente la voluntad de creer que disipaba las dudas de lo contingente; aquí la duda se ha transformado en escándalo e interpela de manera creciente al individuo (EC, p. 153 ss. / SKS 12, p. 128 ss.).

Esta distinción entre una creencia en sentido ordinario y la fe en sentido eminente ha derribado la postura que pretendía ver en Kierkegaard un cristianismo fuertemente volitivo y radicado de manera esencial en el ser humano. Si no se separan los grados de la creencia y la fe, es posible afirmar, como lo hace Louis P. Pojman (1990), que Kierkegaard tiende a una salvación autárquica del hombre sin depender de la gracia, anulando la dialéctica de la redención. Esta dialéctica hace aparecer lo positivo del cristianismo a través de su negatividad, relacionando la posibilidad que se hace presente en la voluntad del hombre y la necesidad que representa para su salvación la imagen de Dios: cuando el individuo se encuentra ante la gracia divina, es cierto que todavía tiene opciones de echarse a un lado y despreciarla, lo que haría parecer que el paso último, y definitivo, se encontraría en lo humano. Sin embargo, esta consideración del problema es meramente positiva y no tiene en cuenta la negatividad dialéctica que reafirma y lleva al extremo de su pasión a la voluntad del individuo en la elección de una sola opción que es verdaderamente elegible (Wahl 1956, p. 54).

Con estos renglones ya avanzados, se nos presenta en Kierkegaard una labor racional en el devenir cristiano quizás más grande de la que esperaríamos encontrar en un pensador que asegura literalmente que la fe está en contra de todo entendimiento ${ }^{5}$ (PS, p. 548 / SKS 7, p. 515). Es más, toda la labor individual que se desarrolla hasta los umbrales de la fe es de índole racional; pero no es una razón cualquiera, sino una razón apasionada y cualitativa, que mantiene siempre en consideración los límites de sus facultades y el ámbito expresamente existencial en el que su tarea es llevada a cabo (Höffding 1930, p. 104). Por eso es que en Temor y temblor (1844), Kierkegaard no ve mella de lo paradójico en lo cristiano cuando habla de los dos movimientos de la fe, la resignación absoluta y el salto mismo de la fe, e incluso ve en el primero, cuyo desarrollo es absolutamente consciente, una condición necesaria para el segundo (TT, p. 64 / SKS 4, p. 140; Munizaga 1956). Abraham, camino al Moriah, sabía que su hijo Isaac moriría en agrado a Dios y comprendía el sacrificio, al tiempo que creía que el mandato era justo y bueno.

en cambio, como ocurre también en nuestra lengua, se puede distinguir con propiedad entre believe y faith (Véase Wisdo 1987, p. 107).

5 Utilizaremos de manera indistinta entendimiento, razón o conciencia, pues en este contexto es indiferente una diferenciación estricta. Lo que interesa notar es la intervención de una facultad de tipo racional humana en el individuo cristiano. 
Terminando con el acto consciente del creyente, y habiendo rozado algunas nociones de la dialéctica cristiana, nos introduciremos ahora en el asunto del espíritu humano y el impacto existencial que tiene para Kierkegaard la conversión cristiana en el individuo.

\section{Las dialécticas del espíritu}

Kierkegaard, todavía enraizado en la terminología hegeliana, define al hombre como espíritu, pero sin apuntar con esto a lo universal, como lo hace el filósofo de Jena, sino que en cada momento a lo particular. Esta constitución espiritual es dada al ser humano como esencia por Dios y solo a través de Él puede ser llevada a acto en la existencia; esta transformación del hombre en espíritu, que le permite ser sí mismo en un sentido auténtico, apoyándose en el poder que lo ha creado, es justamente el devenir cristiano (EM, p. 168 / SKS 11, p. 242). En esta conversión se aparejan contrarios que constituyen en el ser humano las dos naturalezas que encierra su existencia, esto es, lo inmanente y lo trascendente ${ }^{6}$ :

El hombre es espíritu. Mas, ¿qué es el espíritu? El espíritu es el yo. Pero ¿qué es el yo? El yo es una relación que se relaciona consigo misma, o dicho de otra manera: es lo que en la relación hace que ésta se relacione consigo misma. El yo no es la relación, sino el hecho de que la relación se relacione consigo misma. El hombre es una síntesis de infinitud y finitud, de lo temporal y lo eterno, de la libertad y la necesidad, en una palabra: es una síntesis. Y una síntesis es la relación entre dos términos (EM, p. 33 / SKS, p. 129) 7 .

Repasemos, de una a la vez, estas relaciones. La síntesis de lo finito e infinito tiene lugar a partir de un trabajo de conciencia del hombre, y se puede identificar con la resignación absoluta que veíamos más arriba. Al carácter finito e inmediato que tiene todo sujeto en la existencia, se le agrega una porción de infinitud que descubre el individuo en sí mismo a través del interés infinito que adquiere por existir, haciéndose consciente de una porción suya que no corresponde meramente a los límites de la inmanencia. Una vez dada esta dialéctica, que tiene lugar únicamente a nivel de la conciencia, es

6 La influencia socrática en Kierkegaard es siempre evidente, y más en esta división, con raigambre dórica, entre lo referente al cuerpo y lo referente al alma. A pesar de haber criticado antes a Pojman, su rastreo de la influencia socrática es bastante pertinente (1990, p. 58).

7 Por la importancia conceptual de esta cita, la apuntamos también en danés: 'Mennesket er Aand. Men hvad er Aand? Aand er Selvet. Men hvad er Selvet? Selvet er et Forhold, der forholder sig til sig selv, eller er det i Forholdet, at Forholdet forholder sig til sig selv; Selvet er ikke Forholdet, men at Forholdet forholder sig til sig selv. Mennesket er en Synthese af Uendelighed og Endelighed, af det Timelige og det Evige, af Frihed og Nødvendighed, kort en Synthese. En Synthese er et Forhold mellem To'. 
menester que el individuo vuelque todo ese contenido ideal a la realidad, condensando su labor en un cambio real de su existencia ${ }^{8}$. Sin embargo, cualquier esfuerzo que haga el hombre en este punto será en vano, pues la necesidad de lo que ya existe escapa a sus facultades y queda solo como trabajo de Dios, en tanto representa Este la variante de la posibilidad o la libertad. Pero, si es necesaria aquí la ayuda divina, debemos retrotraernos a la dialéctica de la fe que ya teníamos a mano, aunque con los factores invertidos: la necesidad encarnada en la figura de Dios y la posibilidad en la voluntad humana; solo añadiendo esta dialéctica intermedia a las dialécticas del espíritu que son detalladas expresamente por Kierkegaard, es factible ver un avance coherente y entendible del individuo cristiano. Así, habiendo el hombre aunado lo finito y lo infinito en su conciencia, y habiéndolos llevado a la existencia gracias a la dialéctica de la necesidad y la posibilidad, que se da a su vez gracias a la dialéctica de la fe, donde el hombre elige voluntariamente la única opción que se le presenta como verdaderamente elegible, solo entonces es posible que lo temporal y lo eterno se relacionen en el yo a la manera en que estaban relacionados en Cristo: ya siendo espíritu, ya entrando en el devenir del cristianismo, el individuo encarna el paradigma de la verdad de lo humano y llega a ser, por tanto, en esencia y en existencia lo mismo.

De forma paralela y más vivencial, dentro de este marco aparentemente vacío de lo dialéctico, se plasman los tres estadios de la existencia que Kierkegaard reconoce en el ascenso de la conciencia de un individuo: el estético, el ético y el religioso, hallándose entre el primero y el segundo la ironía, y entre el segundo y el tercero el humor (PS, pp. 487-488 / SKS 7, p. 455). El desarrollo se da desde la inmediatez de lo estético hacia un sentido común finito, que es la antesala de lo ético. Así, el individuo se va haciendo consciente de un valor intrínseco en sí mismo -lo que veíamos en la dialéctica de lo finito e infinito-y capta que dentro de la capacidad innata que tiene para comprometerse con su propio existir hay un valor esencial y alejado de la mera inmanencia. Ya en lo ético, el hombre singular se preocupa infinitamente por su interioridad y se encierra en sí mismo, saliendo a la exterioridad solo a través de la ironía. Cuando el hombre ético llega a los umbrales de lo religioso, no se encuentra de lleno y de manera inmediata con lo paradójico-cristiano, sino que entra en una etapa previa que es lo que Kierkegaard llama Religiosidad $A$ o la confirmación dialéctica del individuo interior. Nuevamente, como ocurría con el estadio ético, su forma de aparecer a lo externo será solo a través de un límite específico: el humor. En este punto su noción de que la salvación de su existencia no está en sus manos es plena y reconoce que un poder exterior debe tenderle una mano; según las dialécticas que ya analizábamos, el hombre está aquí a la espera de que ocurra la dialéctica intermedia de la fe. Una vez ocurrida esta, tiene lugar la Religiosidad $B$ o lo paradójico-cristiano, y por primera vez se deja atrás la inmanencia y se trasciende en una relación con lo eterno (PS, p. 538 / SKS 7, p. 506). Por esta razón se dan la paradoja y lo absurdo, pues la salvación eterna de aquel que está deviniendo cristiano se confirma en un punto de la existencia totalmente temporal, asemejándose

8 En Kierkegaard subyace todavía la existencia como un predicado de excelencia y mayor grandeza frente a la pura esencia (EM, p. 35 / SKS 11, p. 131). 
al momento en que Dios introdujo la plenitud en el tiempo al hacerse humano (MF, p. 34 / SKS 4, p. 226). Esto es lo que Kierkegaard llama también el instante (ibíd.), y es a lo que hacíamos alusión con la dialéctica de lo temporal y lo eterno.

Veamos, a modo de breve síntesis, y en palabras de Kierkegaard, un panorama general del ascenso de conciencia del individuo a través de los estadios de la existencia:

Lo inmediato, lo estético, no encuentra contradicción con existir; existir es una cosa, la contradicción es algo distinto que viene de afuera. Lo ético halla contradicción, pero dentro de la autoafirmación. La Religiosidad A comprende la contradicción como sufrimiento en la autoanulación, pero dentro de la inmanencia; pero acentuar éticamente la existencia impide que un existente permanezca abstractamente en la inmanencia o llegue a ser abstracto al querer permanecer en la inmanencia. Lo paradójico-religioso rompe con la inmanencia y hace que existir sea la contradicción absoluta; no dentro de la inmanencia, sino en oposición a la inmanencia. No hay afinidad inmanente que subyazca entre lo temporal y lo eterno, porque lo eterno ha entrado en el tiempo y quiere establecer parentesco ahí (PS, p. 554 ss. / SKS 7, p. 521).

A lo largo tanto de las dialécticas, como de los estadios, lo que marca un cambio en el individuo es un salto cualitativo. Esta imagen la usa Kierkegaard para explicitar la manera singular y apropiadora que debe tener todo proceso individual para configurarse como radical en el hombre. No hay cambios de cualidad que se desplieguen de manera objetiva o de manera general, sino que todo individuo se introduce personal e intransferiblemente en un determinado desarrollo. De esta forma, si la humanidad entera es históricamente pecadora a causa del pecado original, no hay extensión de dicha culpabilidad hacia el individuo que participa en la especie, sino que solo a través de su propia acción puede encarnarse en él el pecado: la humanidad avanza cuantitativamente en el tiempo y el espécimen singular la sigue cualitativamente (CA, p. 66 / SKS 4, p. 335).

Entonces, radicalmente transformado, el individuo cristiano encarna la doctrina y la hace existencia, es salvo y lucha de manera constante con el desafío irresoluble de representar en la imperfección lo perfecto. De esto son marcas la verdad y la libertad.

\section{Verdad y libertad}

Someramente hablando, como nos es consabido, la verdad ha adoptado dos grandes interpretaciones a lo largo de la historia de la filosofía: la aristotélico-tomista y la cartesiana-kantiana (PS, p. 191 / SKS 7, p. 173). La primera habla de una adecuación del ser al pensamiento y la segunda, de una adecuación del pensamiento al ser. Ambas, por ende, buscan dar con el ideal ontológico de la verdad como una igualdad entre el pensamiento y el ser, cosa que acepta intelectualmente Kierkegaard. Sin embargo, el danés pone una traba en cuanto a la certera correspondencia que ostentarían estas visiones tradicionales. La problemática radica en dos puntos: 1) la noción de ser que 
se incluye en los enunciados y 2) en el carácter de existente que posee el sujeto que conoce. Quedémonos con el primer problema.

Si el ser dentro de las dos definiciones de verdad se refiere al ser empírico, esto es, al ser en devenir, lo que se viene abajo es el resultado objetivo que tiene como incentivo el conocimiento tradicional. Dicho de otro modo, la adecuación entre el pensamiento y un ser tal no podría erigirse nunca como definitiva, pues el movimiento inherente al devenir no lo permite: el objeto que se intenta conocer no está todavía terminado y todo se queda en una mera aproximación. Por otro lado, si lo que se tiene por ser en la adecuación es el ser abstracto, es perfectamente factible que se dé un resultado inamovible, precisamente porque la abstracción resta al ser su movimiento. No obstante, es evidente, nos dice Kierkegaard, que una forma tal de conocimiento no representa más que lo que el mismo entendimiento se ha creado para sí como su objeto, mostrando la verdad como una reduplicación completamente abstracta (PS, p. 192 / SKS 7, p. 194) ${ }^{9}$. En resumen, la adecuación empírica se queda en la aproximación objetiva y la adecuación abstracta no es más que una fantasía del pensamiento puro.

Sin embargo, toda verdad o todo conocimiento verdadero que sea ajeno a la existencia y que no esté esencialmente unido a ella es indiferente (PS, p. 199 / SKS 7, p. 181). Lo que se busca, por el contrario, como apuntaba Kierkegaard con el segundo problema de las concepciones tradicionales de la verdad, es dar cabida dentro del ámbito cognoscitivo a un sujeto, que si bien es pensante, es por sobre todo existente. Así, la verdad que únicamente podrá estar atada sustancialmente al hombre será una verdad existencial o, como considera Kierkegaard, la verdad que ha comunicado el cristianismo.

Cristo, a diferencia de lo que marcaba Sócrates con su teoría de la reminiscencia ${ }^{10}$, pone la verdad como externa al ser humano por motivo del pecado original e inalcanzable sin mediar una previa gracia divina, como ocurría también, y no casualmente, en la conformación del espíritu. Cristianamente hablando, no se conoce la verdad, sino que se es la verdad, mostrando en la apropiación y en la reduplicación de lo verdadero una unidad perfecta en lo imperfecto de lo temporal ${ }^{11}$. La verdad es camino y vida, tal como decía Cristo que Él lo era (Juan 14, p. 6):

9 James Collins, primer intérprete serio de Kierkegaard en los Estados Unidos, desarrolla de manera muy satisfactoria, a mi parecer, el problema de la verdad existencial en Kierkegaard, salvo por un pequeño error interpretativo. Él muestra que Kierkegaard rechaza la adecuación idealista del ser al pensamiento, en tanto representa ésta una visión completamente abstracta e irreal, sin percatarse de que el danés se pone en el caso de que ambas interpretaciones contengan el ser en sentido empírico o que ambas lo aborden de manera abstracta, tal como presentamos ahora nosotros. Por lo tanto, lo que considera Kierkegaard como una mera tautología del pensamiento puro es la metodología abstracta de considerar el ser dentro de la adecuación como inmóvil (Collins, 1958, pp. 152-191; Cfr. PS, p. 192 / SKS 7, p. 194).

$10 \quad$ Véase, Platón 2007 y Pojman 1982, p. 58.

11 Véase, Pap., I A 94. 
(...) el ser de la verdad (Sandhedens Veeren) es la duplicación en ti, en mí, en él, de manera que tu vida, la suya, la mía, en el esfuerzo de aproximarnos a ella, exprese la verdad; que tu vida, la suya, la mía, en el esfuerzo de aproximarnos a ella, sea el ser de la verdad, como la verdad era en Cristo: una vida, pues Él era la verdad (EC, p. 206 / SKS 12, p. 202).

La conexión entre fe, espíritu y verdad queda entonces estructurada de la siguiente forma:

1. Según el cristianismo, el hombre fue concebido y creado por Dios como espíritu. El espíritu es la instancia donde una gran síntesis se relaciona consigo misma. Esta síntesis pone uno frente al otro una serie de contrarios que residen dentro del hombre. Sin embargo, éste, a partir del pecado original, perdió el estatuto inmediato de espíritu, de modo que su tarea en la existencia consiste en volver a serlo. Ser espíritu se iguala a la categoría de ser cristiano, lo que se desprende de las enseñanzas de Cristo, que fue enviado por Dios para mostrarle al hombre la medida a la que todos se han de ajustar. En otras palabras, Cristo muestra la verdad de la existencia (EM, p. 148 / SKS 11:225-226).

2. El ser cristiano así concebido da una pauta para un trabajo de conciencia en el hombre, que le ayuda a caer en la cuenta de aquellos contrarios que no se le presentan de forma inmediata: la infinitud, la eternidad y la posibilidad. De esta forma, el hombre se sabe en esencia algo que no es todavía en existencia. Reemplácese esencia por potencia y pensamiento, y existencia por acto y ser.

3. Para ser el hombre aquella síntesis que se relaciona consigo misma, que representa positivamente al espíritu, debe hacer calzar en la existencia los contrarios que residen en él, lo que no puede llevar a cabo sin la ayuda del poder que lo ha creado (Dios). El socorro divino se consigue a través de la gracia y la fe.

4. Teniendo el hombre la gracia y la fe, es capaz de aceptar, en función de su pasión como creyente, el aparejamiento de los contrarios en la existencia. En este punto, el hombre se sabe algo conscientemente y el contenido de esa conciencia es llevado a la existencia por Dios. El hombre es verdad a la medida en que Dios mostró la verdad con Cristo; el hombre encarna la verdad y es como ella ${ }^{12}$.

12 Theodor Haecker, parafraseando a Kierkegaard, dice: "Dios es verdad porque, como subjetividad infinita, está en relación plenamente objetiva con su propia subjetividad y ejecuta de una manera plenamente subjetiva lo que, en su consideración objetiva, ha entendido con respecto a sí mismo, porque puede verse a sí mismo de una manera absoluta y reproducir de un modo absoluta y plenamente subjetivo este momento de sí mismo". En palabras más simples, es en esencia y existencia lo mismo. De un modo similar, aunque 
Ahora, ya habiendo aclarado elementos fundamentales como estos, podemos hacer puente para llegar a la libertad.

Estando el hombre fuera de la verdad o en la no-verdad para el cristianismo, es decir, en el pecado, permanece asimismo sumido en la no-libertad, de la que solo puede liberarlo el conocimiento verdadero o cristiano (CA, p. 243 / SKS 4, p. 439). Como el pecado es una condición en la que cada hombre está por sí mismo, en tanto cada cual introduce cualitativamente el pecado en su vida, se deriva que el apresamiento del que se es víctima no es externo, sino que interno: todo hombre está preso en sí mismo en su cualidad de culpable. De esto podemos servirnos para hablar en Kierkegaard de una libertad como condición o estado -libertad en sentido ordinario-, deslindándola de la libertad como poder -libertad en sentido eminente (Pojman 1990, p. 49).

El libre arbitrio (liberum arbitrium) se puede comparar con la libertad en tanto fuerza volitiva, siendo la sola capacidad no incondicionada de tomar una decisión entre dos o más opciones. Si un inocente, ante la angustia que le genera la posibilidad de su libertad, peca o elige pecar, reafirmará el poder que tiene su voluntad de elegir en dicho acto, aunque se transforme en pecador y yazca irremediablemente en la no-libertad en cuanto estado. Con esta noción de libertad habla Kierkegaard en La enfermedad mortal (1849), cuando dice que el yo es libertad (SKS 11, p. 145) 13, y en la Ejercitación del cristianismo (1850), al apuntar que Cristo busca atraer al hombre solo en cuanto este elige en tanto esencia libre (EC, p. 167 / SKS 12, p. 164). Así, si se une esta concepción de la libertad como poder de elección con la dialéctica intermedia de la fe a la que hemos aludido en reiteradas ocasiones, es posible justificar más nítidamente la diferencia entre dos tipos de libertad patentes en el pensamiento kierkegaardiano.

Cuando se le presenta a un hombre la gracia de la fe, no está todo dicho en cuanto a la transformación de este en cristiano, pues es necesaria todavía la elección libre del individuo frente al don. En esa decisión se aparejan, como bien vimos anteriormente, la posibilidad que representa la voluntad del hombre y la necesidad que significa Dios para hacer del individuo un individuo auténtico. Sin la gracia no hay posibilidad de elección, por lo que lo radical se deja caer en cada caso en las manos de Dios. Mas tampoco se puede hablar de un creyente en sentido pleno si este no da el salto de la fe a través de una decisión consciente. Por esta razón, plantear al yo como libertad antes de transformarse en sí mismo o al hombre como esencia libre ante la atracción de Cristo parece una diferenciación sutil, pero decidora, que separa la posibilidad que significa la libertad en tanto poder, y la posición que representa la libertad en tanto estado.

En la fe, el hombre se vuelve libre: está en la condición de ser libre. La libertad así concebida, eso sí, trae consigo la reducción de la libertad como poder, porque para darse aquélla, a causa de la dialéctica de la gracia, tiene que verse reducida esta. La

todavía sujeto al devenir, el hombre está inmerso en ese esfuerzo de llevar al acto lo que sabe de sí que es en potencia $(1948$, p. 80).

13 Por un error que consideramos meramente casual, el traductor de la edición en español que utilizamos omite este pasaje. 
voluntad del hombre se ve acotada a una sola opción que se erige como verdaderamente elegible y que de no ser elegida supone la permanencia del individuo en la no-libertad ${ }^{14}$. A través del perdón de los pecados que concede la relación con Cristo, el individuo pasa de ser cualitativamente culpable a ser cualitativamente inocente; si el pecado era estar en la no-verdad y esta condición derivaba en la no-libertad, entonces la inocencia traerá consigo la verdad y también la libertad. Donde esté la fe y la condición, ahí estarán también la verdad y la libertad. Con esto podemos entender plenamente al apóstol Juan cuando dice en su Evangelio: "Si permanecéis en mi palabra, seréis en verdad discípulos míos y conoceréis la verdad, y la verdad os liberará” (8, pp. 31-33).

A pesar de haber dejado ciertos elementos de lado, hemos abarcado, en la medida de lo posible, los conceptos y las categorías que parecen de mayor importancia a la hora de delimitar de manera global el cristianismo de Kierkegaard, consignando, a pesar de su escritura seudónima ${ }^{15}$, elementos transversales que dan una pincelada acaso esclarecedora de esta que sería la única explicación consistente de la existencia.

\section{Referencias bibliográficas}

\section{BIBLIOGRAFÍA PRINCIPAL}

Kierkegaard, Søren (1994 -), Søren Kierkegaards Skrifter, edición a cargo del Søren Kierkegaard Forskningscenter, red. Niels Jørgen Cappelørn, Joakim Garff, Anne-Mette Hansen, Jette Knudsen, Johnny Kondrup, Alistair McKinson y Finn Hauberg Mortensen. Copenhague: Gad. Se puede acceder a los tomos publicados en la siguiente página electrónica: www.sks.dk.

(1962-1974), Die Tagebücher, selección, reordenación y traducción de Hayo Gerdes, 5 tomos. Düsseldorf-Köln: Eugen Diederichs Verlag.

(2009), Ejercitación del cristianismo (Indøvelse i Christendom), prólogo y traducción de Demetrio Gutiérrez Rivero. Madrid: Trotta. Abreviatura: EC. (2010), El concepto de la angustia (Begrebet Angest), prólogo, traducción

y notas de Demetrio Gutiérrez Rivero. Madrid: Alianza. Abreviatura: CA.

14 Esto configura al pecado, dentro del cristianismo, como una posición y no como una simple pasividad (CA, p. 239 / SKS 4, p. 436).

15 Tácitamente nos hemos paseado por gran parte de la obra seudónima de Kierkegaard dedicada a la exposición de $s u$ cristianismo, sin hacer diferencia entre los "distintos" autores. Lo hemos hecho así porque salta a la vista, una que se mira la totalidad de lo expuesto, que las discrepancias no superan las concordancias y que, al menos en materia religiosa, Kierkegaard se atrevió a hablar en voz de sus pseudónimos algo más directamente que respecto, por ejemplo, a lo estético o a lo ético. Su obra homónima, en cuanto a su expresa publicación abierta, es mucho más amable para el lector interesado. 
(2008), La enfermedad mortal (Sygdommen til Døden), prólogo y traducción de Demetrio Gutiérrez Rivero. Madrid: Trotta. Abreviatura: EM.

(2009), Migajas filosóficas o un poco de filosofia (Philosophiske Smuler), edición y traducción de Rafael Larrañeta. Madrid: Trotta. Abreviatura: MF.

(2010), Post Scriptum no cientifico y definitivo a las Migajas filosóficas (Afsluttende uvidenskabelig Efterskrift), traducción de Javier Teira y Nekane Legarreta. Salamanca: Sígueme. Abreviatura: PS.

(1975), Temor y temblor (Frygt og Baeven), traducción y notas de Demetrio Gutiérrez Rivero. Madrid: Guadarrama. Abreviatura: TT.

\section{BiBLIOGRAFÍA SECUNDARIA}

Collins, J. (1958), El pensamiento de Kierkegaard, traducción de Elena Landázuri. Buenos Aires: Fondo de Cultura Económica.

Gaye, M. (1987), "Kierkegaard on religious knowledge", History of European ideas, vol. 22, no. 2: 105-112.

Haecker, T. (1948), La joroba de Kierkegaard, traducción del alemán y estudio preliminar por Ramón Roquer. Madrid: RIALP.

Höffding, H. (1930), Søren Kierkegaard, traducción de Fernando Vela. Madrid: Revista de Occidente.

Munizaga, R. (1956), "Filosofía y religión en el pensamiento de Kierkegaard", Revista de Filosofia, vol. III, no. 2: 42-61.

Platón (2007), Menón, en Diálogos, tomo II, introducciones, traducciones y notas de J. Calonge Ruiz, E. Acosta, F. Oliveri y J. L. Calvo. Barcelona: Gredos.

Pojman, L. (1982), "Kierkegaard on faith and history", Philosophy of religion, vol. 13, 57-68.

(1990), "Kierkegaard on faith and freedom", Philosophy of Religion, vol. 27: 41-61.

Sagrada Biblia (1968), traducción de Eloíno Nácar Fuster y Alberto Colugna. Madrid: Biblioteca de Autores Cristianos.

Wahl, J. (1956), Kierkegaard, traducción de José Rovira Armengol. Buenos Aires: Losange.

Wisdo, D. (1987), “Kierkegaard on belief, faith, and explanation”, Philosophy of Religion, vol. 21: 95-114. 